\title{
Detection of Objects by Integrating Watersheds and Critical Point Analysis
}

\author{
G. Fu, S.A. Hojjat, and A.C.F. Colchester \\ Medical Image Computing, Kent Institute of Medicine and Health Sciences, \\ University of Kent, Canterbury, Kent, CT2 7PD, UK \\ \{gf23, S.A.Hojjatoleslami, A.Colchester\}@kent.ac.uk
}

\begin{abstract}
This paper presents an improved method for detection of "significant" low-level objects in medical images. Information derived from watershed regions is used to select and refine saddle points in the discrete domain and to construct the watersheds \& watercourses (ridges and valleys). The method overcomes previous topological problems where multiple redundant saddle points are detected in digital images. We also demonstrate an improved method of pruning the tessellation from which salient objects are defined. Preliminary evaluation was based on theoretical analysis, visual inspection of a set of medical images, and human observer experiments with promising result.
\end{abstract}

\section{Introduction}

Ridges and valleys have been usually used to describe and characterize geometry of an object. Like other data-driven techniques, ridges and valleys produce useful lowlevel information which can be used in a large variety of higher level image analysis applications such as image segmentation and registration. Griffin used ridges/valleys to segment angiogram and MR images [4]. Gauch applied the intensity axis of symmetry to segment DSA, CT images and argued that better segmentation could be obtained by using global information provided by image shape analysis [3]. Intensity ridges/valleys of intensity have also been used for blood vessel segmentation where they tend to appear at the centers of blood vessels [2]. Ridges/valleys have proved to be viable for registration for multimodality medical images in many papers [5].

The ridges/valleys of the original or zero order image, as opposed to a gradient magnitude or first order images, can be used to extract the main structure of objects even without locating their boundaries. In medical images, some applications require the detection of objects in very low contrast areas with blurred or weak boundaries. The ridges/valleys work well for detecting these as well as higher contrast objects. There are many different definitions of ridges/valleys in the literature. Our work is based on the definition of separatrices formulated by Nackman in terms of slope districts [6]. The ridge/valley lines are defined as the ascending/descending slope lines emanating from saddle points. The dales/hills are defined as districts whose lines of slope run to/come from the same pit/peak.

It is simple to extend the definitions of maxima and minima from the continuous domain to the discrete domain. However, saddle definition in the discrete domain encounters topological problems. Saddle points are usually defined in terms of an 
analysis of the change of the radial gradient covering the complete neighbourhood. Griffin [4] defined saddles as points having four or more zero crossing in the radial gradient function. We refer to this as the "4+ zero crossing" definition. Rosin concluded that the definition using 4-neighourhood system identifies insufficient saddle points and using the 8-neighourhood system identifies too many saddle points [7]. The latter may detect two adjacent saddle points at least one of which is redundant. Adjacent saddle points generate duplicate or crossing uphill/downhill paths. We demonstrate this problem using a digitized continuous function $f(x, y)=x^{2}-y^{2}+x y$ shown in Fig.1(a). The point at $(0,0)$ is identified as a saddle point by its Hessian Matrix. We sampled the continuous surface at different resolution and detected saddles using the " $4+$ zero crossing" definition with 8 neighbours. The number of detected saddles for four different sampling resolutions was 5, 4, 2, and 1 (Fig.1 b). Several studies have been attempted to solve the problem by triangulation in order to satisfy the topological requirements. Scott showed that triangulation indeed satisfied the topological criteria [8], but different triangulation methods will generate different relationships between pixels, and produce different saddle points and, therefore, different results. Here we present a simple multistage process using local regional information to solve the adjacent saddle point problem in digital images without triangulation.

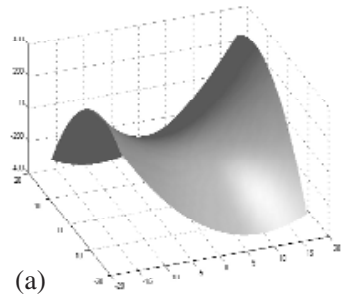

(b)
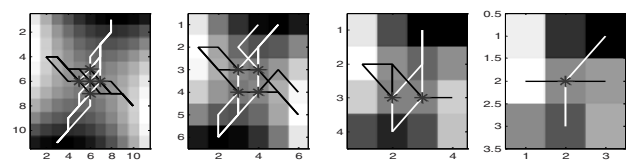

(c)
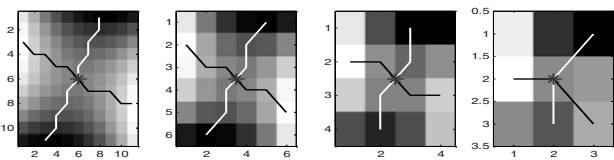

Fig. 1. Adjacent saddle point problems encountered with 8-neighourhood connectivity. The original surface $f(x, y)=x^{2}-y^{2}+x y$, (a) is sampled at 4 different resolutions. (b) saddle points detected by the " $4+$ zero crossing" definition. (c) saddle points detected by our algorithm. The dark dots are saddle points; ridge lines are black and valley lines are white.

The ridges and valleys form a dense network which connects all the structures in an image. This network needs simplification to identify significant ridges and valleys. A bright object can be represented by an isolated maximum or multiple maxima linked by "significant" ridges. A dark object can be represented by an isolated minimum or multiple minima linked by "significant" valleys. Griffin proposed a simple criterion to extract the "significant" ridge and valley subset based on 5 critical point values and the length of their MGPs [4]. Our initial analysis suggested that performance would be improved if the values were derived from local "support region" rather than point values and we present a new and improved method. 


\section{Methods}

To solve the adjacent saddle point problem, we combine a watershed algorithm with critical point analysis (fig. 2).

We apply a watershed algorithm to the original image and its inverse image. Each minimum is related to a dale region and each maximum to a hill region. The set of border points of the regions is defined by $\partial X=\{<s, t>\mid s \in N(t) \cap L(s) \neq L(t)\}, \quad$ where $N(t)$ refers to the 8 neighbours of $t$ and $L$ is the label of regions. For any pair of border pixels between dale regions, the one with higher grey level is selected as a ridge candidate, $R c=\left\{s \mid<s, t>\in \partial X_{d}, \operatorname{im}(s) \geq \operatorname{im}(t)\right\}$, where the $\partial X_{d}$ is the set of border points of the dale regions. For any pair of border pixels between hill regions, the one with lower grey

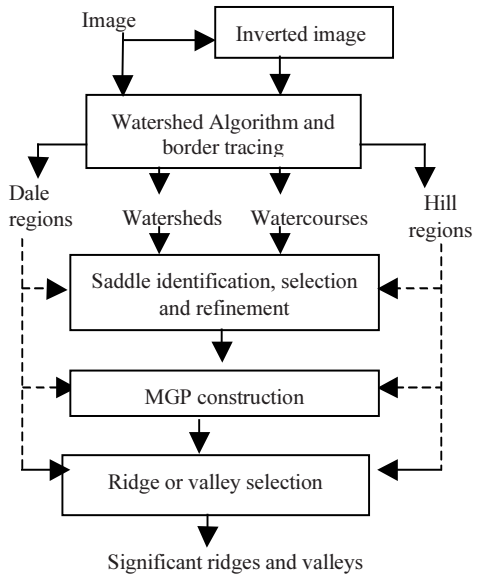

Fig. 2. Outline of the algorithm level is selected as a valley candidate $V c=\left\{s \mid<s, t>\in \partial X_{h}, \operatorname{im}(s) \leq \operatorname{im}(t)\right\}$, where the $\partial X_{h}$ is the set of border points of the hill regions. There are several efficient morphological watershed algorithms. Here, we use Bieniek’s algorithm [1].

\subsection{Saddle Candidate Identification, Selection, and Refinement}

We define the saddle candidates $S_{c r}$ on ridges and $S_{c v}$ on valleys as follows:

$$
\begin{array}{ll}
S_{c r}=\left\{p_{i} \in R c \mid \exists \alpha, \beta, \gamma, \delta \in[0,2 \pi)\right. & \left.g_{i}(\alpha)=g_{i}(\beta)=g_{i}(\gamma)=g_{i}(\delta)=0\right\}, \text { and } \\
S_{c v}=\left\{p_{i} \in V c \mid \exists \alpha, \beta, \gamma, \delta \in[0,2 \pi)\right. & \left.g_{i}(\alpha)=g_{i}(\beta)=g_{i}(\gamma)=g_{i}(\delta)=0\right\},
\end{array}
$$

where $g_{i}$ is the radial gradient function.

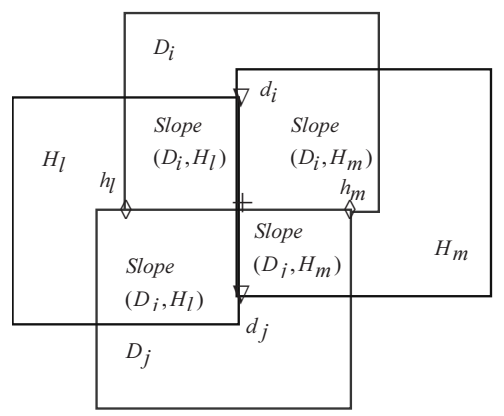

Fig. 3. Slope districts formed by hill regions and dale regions
Typically, a saddle point connects two minima and two maxima whose related dale and hill regions form four slope districts as shown in Fig. 3. $D_{i}$ and $D_{j}$ are dale regions related to two minima $d_{i}$ and $d_{j}$, and $H_{l}$ and $H_{m}$ are hill regions related to two maxima $h_{l}$ and $h_{m}$. The four-slope-district group consists of Slope $\left(D_{i}, H_{l}\right), \quad$ Slope $\left(D_{i}, H_{m}\right)$, Slope $\left(D_{j}, H_{l}\right)$ and $\operatorname{Slope}\left(D_{j}, H_{m}\right)$. The borders of $D_{i}$ and $D_{j}$ are watersheds or 
ridge candidates, while the borders of $H_{l}$ and $H_{m}$ are watercourses or valley candidates. Ridges and valleys cross at saddle points. The watersheds and watercourses within the four-slope-district group should have only one intersection point. Therefore, only one saddle should be identified, and its uphill and downhill paths lead to $h_{l}, h_{m}$ and $d_{i}, d_{j}$ respectively. However, the "4+ zero crossing" definition of saddles in the discrete domain may detect more than one saddle in a four-slope-district group. We introduce the following rules which are applied in a group of four adjacent slope districts to solve the adjacent saddle point problem.

Rule 1: If there is only one saddle candidate (i.e. one point which is both a ridge and a valley saddle candidate), then this point is accepted as a true saddle.

Rule 2: If there is more than one saddle candidate which is both a ridge and a valley saddle candidate, then the saddle candidate whose uphill path and downhill paths intersect at an angle closest to 90 degrees is selected.

Rule 3: Ridge and valley lines may cross at a crack between pixels. If more than one saddle candidate exists in the four-slope-district neighbourhood, but none of them is both a ridge saddle candidate and a valley saddle candidate, then two adjacent saddles (one on a ridge and the other in a valley) will be substituted by a new saddle point, located at the position where the ridge saddle's uphill path crosses with the valley saddle's downhill path.

\subsection{Selection of "Significant" Ridges or Valleys}

The key stage in defining objects is deciding at each saddle point whether it lies on a significant ridge (in which case its uphill paths are preserved and downhill paths are suppressed) or in a valley (in which case the opposite is carried out). We apply criteria in three stages to make this choice. Level 1 criteria use the information from the dale and hill regions related to the saddle (Fig. 3); the level 2 criteria use the information from a four-slope-district group; and the level 3 criteria use only the information from the related maxima and minima. As we move from level 1 to level 3 , the decision is made hierarchically from larger to smaller local regions. If in level 1 , no decision is made; the decision will be made at level 2 or failing that at level 3 . The criteria are formally expressed in table 1.

Level 1 criteria (hill and dale region information): The uphill paths are selected as "significant" ridge lines, if a saddle's value is higher than the mean value of the four regions and also higher than the mean value of each dale region. The downhill paths are selected as "significant" valley lines, if a saddle's value is lower than the mean value of the four regions and also lower than the mean value of each hill region. If no decision is made, then level 2 criteria are applied.

Level 2 criteria (slope district information): The uphill paths are selected as "significant" ridges, if a saddle's value is higher than the mean value of the slope districts in each dale region. The downhill paths are selected as "significant" valleys, if a saddle's value is lower than the mean value of the slope districts in each hill region.

Level 3 criteria (critical point information): The uphill paths are selected as "significant" ridge lines, if a saddle's value is greater than the mean value of the 
maxima and minima. The downhill paths are selected as "significant" valley lines, if a saddle's value is lower than the mean value of the maximum and minimum.

Table 1. The three level criteria for the slection of significant ridges and valleys

\begin{tabular}{|c|c|c|}
\hline Level & Significant Ridge conditions & Significant Valley conditions \\
\hline 1 & $\begin{array}{l}s>\text { mean }\left(i m\left(D_{i} \cup D_{j} \cup H_{l} \cup H_{m}\right)\right) \\
\cap s>\text { mean }\left(i m\left(D_{i}\right)\right) \cap s>\text { mean }\left(i m\left(D_{j}\right)\right)\end{array}$ & $\begin{array}{l}s<\text { mean }\left(\operatorname{im}\left(D_{i} \cup D_{j} \cup H_{l} \cup H_{m}\right)\right) \\
\cap s<\text { mean }\left(\operatorname{im}\left(H_{l}\right)\right) \cap s<\text { mean }\left(\text { im }\left(H_{m}\right)\right)\end{array}$ \\
\hline 2 & $\begin{array}{l}s>\text { mean }\left(\operatorname{im}\left(D_{i} \cap\left(H_{l} \cup H_{m}\right)\right)\right) \\
\cap s>\text { mean }\left(i m\left(D_{j} \cap\left(H_{l} \cup H_{m}\right)\right)\right)\end{array}$ & $\begin{array}{l}s<\text { mean }\left(i m\left(H_{l} \cap\left(D_{i} \cup D_{j}\right)\right)\right) \\
\cap s<\text { mean }\left(i m\left(H_{m} \cap\left(D_{i} \cup D_{j}\right)\right)\right)\end{array}$ \\
\hline 3 & $s \geq\left(i m\left(d_{i}\right)+i m\left(d_{j}\right)+i m\left(h_{l}\right)+i m\left(h_{m}\right)\right) / 4$ & $s<\left(i m\left(d_{i}\right)+i m\left(d_{j}\right)+i m\left(h_{l}\right)+i m\left(h_{m}\right)\right) / 4$ \\
\hline
\end{tabular}

\section{Results}

Our algorithm can be applied to any kind of image. No parameter requires adjustment. We have evaluated the performance on MR images of the head (Fig. 5), X ray angiograms (Fig. 7) and mammograms.

\subsection{Solution of the Adjacent Saddle Point Problem}

In order to illustrate adjacent saddle point problem, we chose a small region of interest shown in Fig.4 from the MR image shown in Fig. 5. Using the common "4+ zero crossing" definition, five saddle points were detected. Saddle points 1, 2 and 3, 4 are two pairs of adjacent saddle points which were located in the same set of four slope districts and thus their uphill and downhill paths lead to the same maxima and minima. The uphill paths of the saddle point 5 did not lead to its two related maxima. Fig.4 (c) illustrates the result of our algorithm. The adjacent saddles were refined. As expected, the uphill paths of saddle 5 were correctly connected to its related maxima.

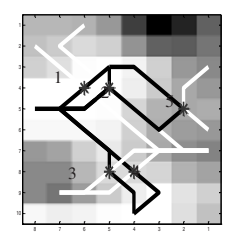

a

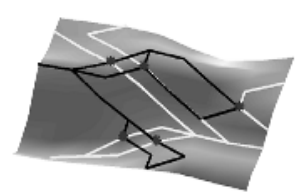

$\mathrm{b}$

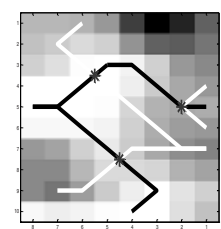

$\mathrm{c}$

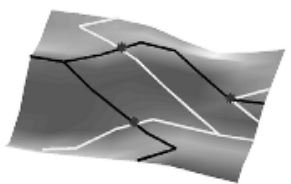

d

Fig. 4. The critical points detected using " $4+$ zero crossing" is shown on the original image (a) and on a perspective view of the grey scale surface (b). The result of our algorithm is shown in (c) and (d). The stars are saddle points; ridge lines are black and valley lines are white.

One topological property of critical points is formalized by the Euler criterion: $\mathrm{H}+\mathrm{V}-\mathrm{S}=2(1-\mathrm{g})$, where $\mathrm{H}$ is the number of peaks, $\mathrm{V}$ is number of pits, $\mathrm{S}$ is the number of saddles, and 2(1-g) is Euler number [8,9]. We applied the "4+ zero crossing" 
definition and our algorithm to detect saddles for three different kinds of images. The results are shown in table 2 . Using the " $4+$ zero crossing" method, a large number of spurious saddle points were detected. By applying our algorithm, the final number is close to the estimated number of saddles according to the Euler criterion. We noticed that our algorithm detected fewer saddles than the Euler number for mammogram image. This appears to be due to the fact that in mammogram image there are many high frequency changes associated with "monkey" or higher order saddles.

Table 2. Saddle detection by the "4+ zero crossing" definition and our algorithm.

\begin{tabular}{|l|l|l|l|l|l|l|l|l|}
\hline Image & $\begin{array}{l}\text { Size } \\
\text { (pixels) }\end{array}$ & $\begin{array}{l}\text { Number of } \\
\text { maxima }\end{array}$ & $\begin{array}{l}\text { Number of } \\
\text { minima }\end{array}$ & $\begin{array}{l}\text { Predicted } \\
\text { number } \\
\text { saddles* }\end{array}$ & $\begin{array}{l}\text { Saddles detected by "4+ } \\
\text { zero crossing" }\end{array}$ & $\begin{array}{l}\text { Saddles detected by } \\
\text { our algorithm }\end{array}$ \\
\hline Angiogram & $610 \times 500$ & 2744 & 2837 & 5579 & 24666 & 19087 & 5679 & 100 \\
\hline MRI & $256 \times 256$ & 425 & 422 & 845 & 2414 & 1569 & 852 \\
\hline Mammogram & $203 \times 298$ & 1350 & 1265 & 2613 & 5321 & 2708 & 2558 \\
\hline
\end{tabular}

* Euler Criterion, $\mathrm{H}+\mathrm{V}-\mathrm{S}=2$, is used to estimate the number of saddles. It assumes that all saddle points are standard saddles surrounded by two higher regions and two lower regions [8]. If a monkey saddle or higher order saddle is encountered then the formula to estimate the number of the saddles has to be modified accordingly [8]. ** Difference from Euler criterion.

\subsection{Object Detection by Selection of "Significant" Ridges and Valleys}

Fig.5 (a) is a T2-weighted MRI of the head showing lesions in multiple sclerosis. Fig.5 (c) shows the "significant" ridges/valleys selected by our criteria. A region of interest of fig.5 is shown in Fig. 6. High signal objects, which include grey matter \& scalp, are defined by a closed valley loop surrounding them, and contain one or more local maxima. In this application, these objects include the MS lesions (objects 3-10), grey matter (objects 2 and 11-13) and 1 (scalp). The objects vary greatly in size and shape but all are detected successfully without any modification of parameters. Fig.7 (a) illustrates a region of interest of an x-ray angiogram image. A very high proportion of the blood vessels are detected as significant ridges shown in Fig. 7 (b).

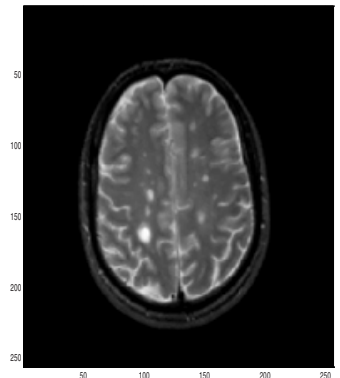

a

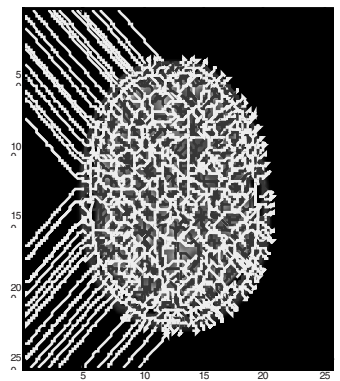

$\mathrm{b}$

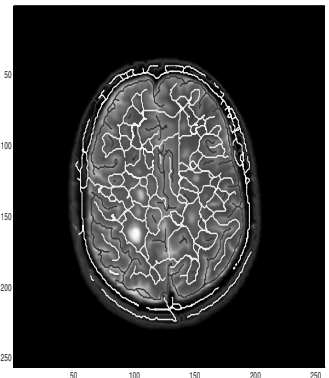

$\mathrm{c}$

Fig. 5. (a) A MR image of head. (b) The constructed ridges and valleys (c) The "significant" bright objects identified by our method. Ridge lines are black and valley lines are white.

Our method is data-driven and is aimed at detecting primitive objects in any type of images. These low level objects may correspond directly to domain-specific objects (e.g. blood vessels) or may be subject to grouping or splitting processes 
according to requirements of different applications. Partly for these reasons, evaluation of generic low-level methods is particularly difficult. We have used human visual judgment of local features as a standard for comparison. A restricted field of view containing a specific saddle and its related maxima \& minima, which limited domain-specific judgments, was used. The subject was asked to decide if the saddle seems to belong to adjacent dark areas or to adjacent light areas. The experiments were performed for all saddle points in the MR image of Fig.5. Table 3 shows the agreement between four observers and the two computer methods. The average interobserver agreement for the observers was $90 \%$. Compared to Griffin's algorithm, the new algorithm showed improved agreement for all observers.

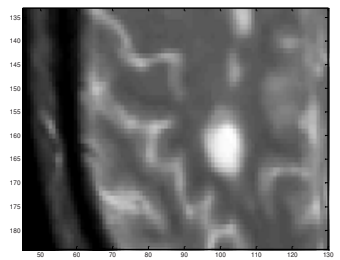

a

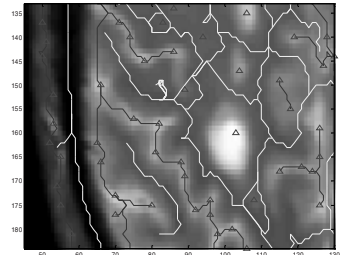

b

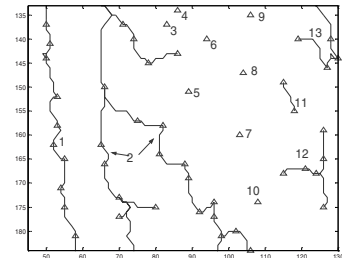

c

Fig. 6. (a) Region of interest from fig.5. (b) The significant ridges \& valleys of (a). (c) The labels denoting the bright objects, which are identified by either an isolated maximum, or multiple maxima linked by "significant" ridges. The maxima are marked by triangles.

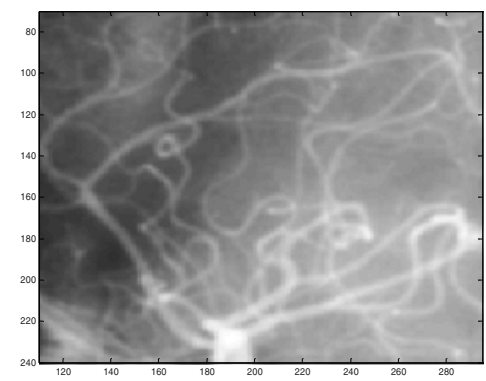

a

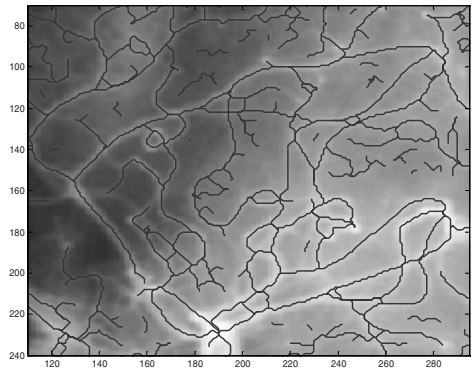

$\mathrm{b}$

Fig. 7. (a) Region of interest of angiogram image (b) the significant ridges of (a)

Table 3. The cross comparison table of agreements between observers and computer methods.

\begin{tabular}{|l|c|c|c|c|c|}
\hline & Our algorithm & Griffin's Criterion & Observer 1 & Observer 2 & Observer 3 \\
\hline Observer 1 & $89 \%$ & $87 \%$ & & & \\
\hline Observer 2 & $88 \%$ & $86 \%$ & $91 \%$ & & \\
\hline Observer 3 & $93 \%$ & $88 \%$ & $89 \%$ & $89 \%$ & $92 \%$ \\
\hline Observer 4 & $91 \%$ & $87 \%$ & $90 \%$ & $92 \%$ & \\
\hline Average of 1-4 & $90 \%$ & $87 \%$ & & & \\
\hline "G. T." * & $94 \%$ & $91 \%$ & & & \\
\hline
\end{tabular}

*The "Ground Truth" is the subset of saddles (93.4\% of total) for which at least 3 observers agreed on the ridge or valley classification. 


\section{Discussion and Conclusions}

In digital images, the definition of saddle points frequently encounters topological problems, which we refer to as the "adjacent saddle point problem". In this paper, we presented an algorithm to solve the adjacent saddle point problem by combining watersheds with the analysis of the relationship between critical points. Our algorithm selects and refines adjacent saddle points using three simple rules based on regional information provided by the watershed algorithm. We also used the watersheds/watercourses to construct uphill/downhill paths.

Another important focus of this paper is on the selection of "significant" ridges and valleys of the original image. The "significant" ridge and valley lines represent the core or middle structure of objects. We defined 3 level criteria from large to small local region. Initial evaluation using four independent observers shows that the selection of "significant" ridges/valleys is performed very close to human judgment.

The concepts presented here extend naturally to first order (gradient magnitude) images where ridges correspond to edges. However, the present work specifically on zero order images where object detection can be successfully accomplished independently of edge localization. Our method can detect very dim objects in very low contrast areas. There are many clinical applications where this is potentially useful, particularly where edge contrast tends to be variable or controversial. MS lesion detection is one such application.

Acknowledgements. The work was supported in part by the EC-funded projects QAMRIC (BMH4-98-6048) and PVEOUT (2000-00594).

\section{References}

1. Bieniek, A., "An efficient watershed algorithm based on connected components," Pattern Recognition, vol. 33 pp. 907-916, 2000.

2. Chen, Z. and Molloi, S., "Vascular tree object segmentation by deskeletonization of valley courses," Computerized Medical Imaging and Graphics, vol. 26 pp. 419-428, 2002.

3. Gauch, J. M. and Pizer, S. M., "The Intensity Axis of Symmetry and Its Application to Image Segmentation" IEEE Transaction on PAMI, vol. 15, no. 8, pp. 753-770, 1993

4. Griffin, L. D., Colchester, A. C. F., and Robinson, G. P., "Scale and segmentation of greylevel images using maximum gradient paths," Image and Vision Computing, vol. 10, no. 6, pp. 389-402, 1992

5. Lopez, A. M, "Multilocal Methods for Ridge and Valley Delineation in Image Analysis." $\mathrm{PhD}$ thesis, the Universitat Autonoma de Barcelona, 2000.

6. Nackman, L. R., "Tow-Dimensional Critical Point Configuration Graphs," IEEE Transaction on PAMI, vol. 6, no. 4, pp. 442-450, 1984.

7. Rosin, P. L., "Early Image Representation by Slope Districts," Journal of Visual Communication and Image Representation, vol. 6, no. 3, pp. 228-243, Sept.1995.

8. Scott, P., "An Algorithm to Extract Critical Points from Lattice Height Data," International Journal of Machine Tools and Manufacture, vol. 41 pp. 1889-1897, 2001.

9. Stewart, I., "A Swift Trip over Rugged Terrain-Mathematical recreations," Scientific American, pp. 89-91, June1991. 